NOTA CIENTFICA

\title{
Análise visual de pimentão verde armazenado sob temperatura controlada
}

\section{Visual analysis of green peppers stored under controlled temperature}

\author{
Ana Marinho do Nascimento $*^{1}$, Franciscleudo Bezerra da costa ${ }^{2}$; Jéssica Leite da Silva ${ }^{3}$, Mahyara de Melo Santiago ${ }^{4}$, \\ Larissa de Sousa Sátiro 5
}

\begin{abstract}
Resumo: O pimentão (Capsicum annuum. L.), é uma hortaliça extremamente perecível, que deteriora facilmente gerando elevados índices de perdas após sua colheita. A aceitação dos produtos hortícolas no mercado depende da análise visual, sendo a primeira ocorrência no momento da compra. Para identificar frutos e hortaliças de qualidade é necessário observar alguns atributos sensoriais, que são indispensáveis na confirmação da qualidade. Objetivou-se avaliar parâmetros como aparência, odor, cor e perda de massa de pimentão verde por um período de 15 dias, identificando o tempo aproximado de estabilidade dessa hortaliça na temperatura controlada. Foram utilizados cerca de $5 \mathrm{~kg}$ de pimentões verdes provenientes no Centro Econômico de Abastecimento Sociedade Anônima, localizada na cidade de Patos, Paraíba. Os pimentões foram selecionados e lavados em água corrente. Os mesmos foram pesados e colocados em bandeja de poliestireno sem revestimento, emparelhados sob uma bancada e mantidas na temperatura controlada $\left(24 \pm 1^{\circ} \mathrm{C}\right)$. Os pimentões mudaram a sua coloração no decorrer da análise, passando de verde brilhante para metade verde e metade marrom. Os pimentões verdes sofreram modificações visuais no decorrer do armazenamento, a estabilidade dessa hortaliça na temperatura controlada foi de aproximadamente 6 dias.
\end{abstract}

Palavras-chave: Capsicum annuum. L.; Perda de massa; Perecível; Senescência.

\begin{abstract}
The Chili (Capsicum annuum. L.), is an extremely perishable vegetable, which deteriorates easily, resulting in high losses after harvesting. The acceptance of vegetables products in the market depends on the visual analysis, being the first occurrence at the time of purchase. To identify quality fruits and vegetables it is necessary to observe some sensorial attributes, which are indispensable in confirming quality. The objective was to evaluate parameters such as appearance, odor, color and mass loss of green peppers for a period of 15 days, identifying the approximate time of stability of this vegetable at controlled temperature. About $5 \mathrm{~kg}$ of green peppers were used at the Centro Econômico de Abastecimento Sociedade Anônima, located in the Patos city, Paraíba. The peppers were selected and washed in running water. They were weighed and placed in uncoated polystyrene tray, paired under a bench and maintained at controlled temperature $\left(24 \pm 1{ }^{\circ} \mathrm{C}\right)$. The chilies changed their color throughout the analysis, going from bright green to half green and half brown. The green peppers underwent visual modifications during the storage, the stability of this vegetable at the controlled temperature was approximately 6 days.
\end{abstract}

Keywords: Capsicum annuum. L.; Weight loss; Perishable; Senescence.

\footnotetext{
*Autor para correspondência

Recebido para publicação em 08/08/2016; aprovado em 25/10/2016

${ }^{1}$ Doutoranda em Engenharia de Processos, UFCG, Câmpus Campina Grande-PB, e-mail: anamarinho06@hotmail.com

${ }^{2}$ Professor do Centro de Ciências e Tecnologia Agroalimentar, UFCG, Câmpus Pombal-PB, e-mail: franciscleudo@yahoo.com.br

${ }^{3}$ Doutoranda em Engenharia de Processos, UFCG, Câmpus Campina Grande-PB, e-mail: jessicaleite2010@gmail.com

${ }^{4}$ Engenheira de Alimentos, UFCG, Câmpus Pombal-PB, e-mail: mahyaramelo16@ hotmail.com

${ }^{5}$ Graduada em Ciências Biológicas, UFCG, Câmpus de Patos-PB, e-mail: lariisatiro@ gmail.com
} 


\section{INTRODUÇÃO}

O pimentão (Capsicum annuum. L.), pode ser classificado como um fruto tipo baga, ele possui um pericarpo carnoso e um epicarpo de cor verde, que ao completa a sua maturação torna-se colorido. É um fruto oco de sementes brancas, achatadas ligadas a um cordão existente no interior do fruto (FILGUEIRA, 2003).

O plantio inicial dessa hortaliça é realizado em bandejas para posteriormente ser transplantado em local permanente. É uma espécie de origem tropical, podendo ser melhor cultivada numa faixa de temperaturas entre $15^{\circ}$ a $25^{\circ} \mathrm{C}$. A sua produção em ambiente protegido tem aumentado devido o pimentão ser uma planta que não tolera frio. Nas regiões onde o inverno é ameno ele pode ser produzido durante $\mathrm{o}$ ano todo (EMBRAPA/SEBRAE, 2010).

É uma hortaliça originária do continente americano que pode ser cultivado em regiões quentes como as do Nordeste brasileiro (MONTEIRO et al., 2009). O pimentão é um fruto que pode ser comercializado verde ou maduro, ou seja, ele é colhido de acordo com a preferência do mercado consumidor e logística de entrega, sendo a vida pós-colheita menor quando os frutos são colhidos no completo estádio de maturação (EMBRAPA/SEBRAE, 2010). No mês de maio de 2016, o pimentão esteve dentro de um grupo de hortaliças que registraram uma redução média de preços, com percentuais entre $15 \%$ e $35 \%$ (CONAB, 2016).

É uma hortaliça extremamente perecível, que se estraga facilmente gerando elevados índices de perdas após sua colheita. Os prejuízos iniciam no campo e são provenientes da ação de patógenos, questões fisiológicas e alguns fatores físicos que ocorre durante o transporte e armazenamento (ANUÁRIO, 2012).

Existem alguns distúrbios fisiológicos que afetam os frutos após a colheita, eles são ocasionados por fatores não parasitário e gera sabores anormais nos frutos. Entre eles encontrasse: estádio de maturação, tempo entre a colheita e refrigeração e condições de armazenamento (CANTILLANO; GIRARDI, 2017).

A aceitação dos produtos hortícolas no mercado depende de parâmetros como cor e aroma, a análise visual é o primeiro acontecimento que ocorre no momento da compra, sendo uma característica decisiva na escolha e aceitação do produto (LIMA et al., 2007). Portanto para identificar frutos e hortaliças de qualidade é necessário observar algumas características como aparência, aroma e cor. Esses atributos são extremamente importantes para manter as propriedades da matéria prima e são indispensáveis na manutenção da qualidade no período de comercialização (CHITARRA; CHITARRA, 2005).

O objetivo do presente estudo foi avaliar parâmetros como aparência, aroma, cor e perda de massa de pimentão verde por um período de 15 dias identificando o tempo aproximado de estabilidade dessa hortaliça na temperatura controlada.

\section{MATERIAL E MÉTODOS}

Foram utilizados cerca de $5 \mathrm{~kg}$ de pimentões verdes provenientes no Centro Econômico de Abastecimento Sociedade Anônima (CEASA), localizada na cidade de Patos, Paraíba. Os pimentões foram acondicionados em caixas plásticas e transportados para o Laboratório de Química,
Bioquímica e Análise de Alimentos, Centro de Ciências e Tecnologia Agroalimentar da Universidade Federal de Campina Grande, Câmpus Pombal.

Os pimentões foram selecionados visualmente, descartando-se aqueles com injúrias, defeituosos, atacados de pragas e com podridões (Figura 1). Posteriormente, foram lavados em água corrente removendo as sujidades superficiais, sendo secados naturalmente.

Figura 1. Pimentões verde selecionados para análise visual, Pombal, Paraíba.

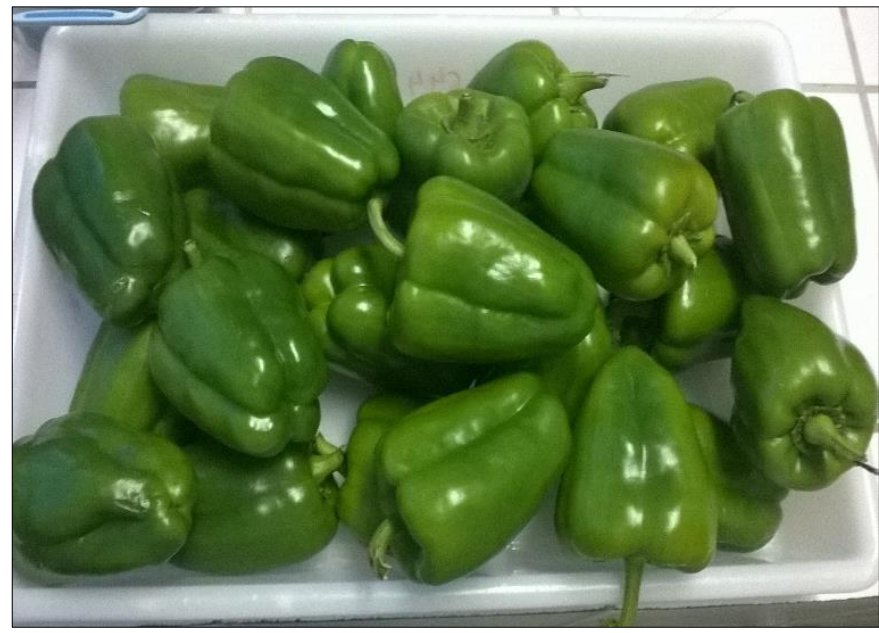

Fonte: Autor Principal (2016)

A Figura 2 descreve em detalhes o fluxograma de aquisição das amostras para análise visual. No qual os frutos foram pesados e colocados individualmente em bandeja de poliestireno sem revestimento, posteriormente foram emparelhados sob bancada e mantidas sob temperatura por meio de ar condicionado $\left(24 \pm 1{ }^{\circ} \mathrm{C}\right)$.

Figura 2. Fluxograma de preparo das amostras de pimentão verde para análise visual, Pombal, Paraíba.

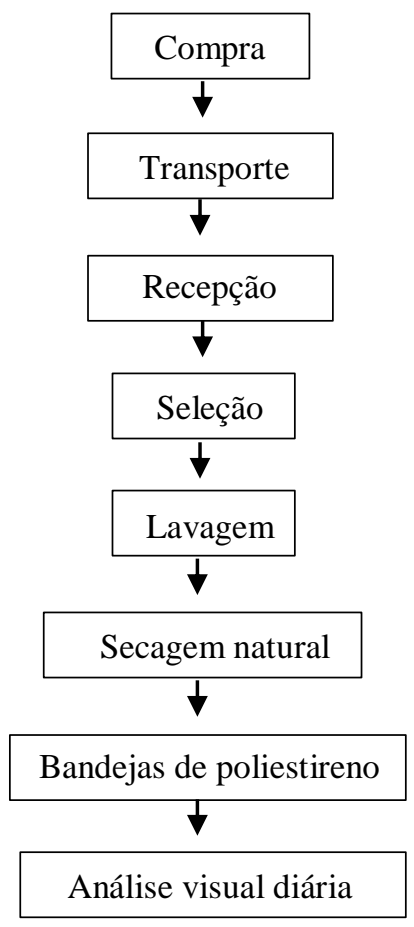


As análises de aparência, cheiro e cor foram realizadas todos os dias as 7 horas da manhã, sendo verificada pelo o mesmo analista durante um período de 15 dias.

A massa fresca foi medida utilizando-se balança semianalítica digital da marca (Bel) com capacidade de $600 \mathrm{~g} \mathrm{e}$ resolução $0,1 \mathrm{~g}$ durante o período um período de 15 dias. $\mathrm{O}$ procedimento foi interrompido, quando os pimentões já não apresentavam condições de consumo, já entrando no processo de senescência. As médias dos dados obtidos foram calculados no software Microsoft Excel 2013.

\section{RESULTADOS E DISCUSSÃO}

Os pimentões mudaram a sua coloração no decorrer da análise, passando de verde brilhante (Figura 3A) para verde opaco com poucas rugas (Figura $3 \mathrm{~B}$ ), seguindo para metade verde opaco, metade marrom e com poucas rugas (Figura
3C). No final da análise os frutos se tornaram enrugados, murchos e sem firmeza (Figura 3D).

De acordo com Albieri (2005), essas ocorrências podem esta relacionadas com a transpiração, visto que, os vegetais possuem cerca de 85 a $95 \%$ de água, como no ambiente a umidade é menor, uma parte da água livre presente nos vegetais é perdida para o ambiente. Esse fator quando em excesso, gera modificações na aparência dos produtos hortícolas, tornando-os enrugados, opacos, flácidos e murchos, além de diminuir o seu peso.

Assim verifica-se que os pimentões são como frutos não-climatéricos, ou seja, após a colheita os mesmos sofreram algumas alterações, porém não amadurecem (Figura 4). Segundo Morgado et al. (2008), para evitar o murchamento dessa hortaliça é necessário reduzir a taxa de transpiração, isso pode ser feito atravéis do aumento da umidade e redução da temperatura.

Tabela 1. Representação visual diária do pimentão verde durante o período de 15 dias de armazenamento à temperatura controlada, Pombal, Paraíba.

\begin{tabular}{ccccc}
\hline Dias & & Características & & \\
\cline { 2 - 5 }${ }^{\circ} \mathrm{C}$ & Aparência & Cor & Aroma & Perda de massa $(\%)$ \\
\hline 0 & Pele lisa e atrativa & Verde brilhante & Forte & $0,0 \pm 0,0$ \\
1 & Pele lisa e atrativa & Verde brilhante & Forte & $1,8 \pm 0,2$ \\
2 & Pele lisa e atrativa & Verde brilhante & Forte & $3,7 \pm 1,3$ \\
3 & Pele lisa e atrativa & Verde brilhante & Forte & $4,9 \pm 0,4$ \\
4 & Poucas rugas & Verde opaco & Forte & $7,2 \pm 0,6$ \\
5 & Poucas rugas & Verde opaco & Forte & $8,6 \pm 0,7$ \\
6 & Poucas rugas & Verde opaco & Forte & $11,0 \pm 1,1$ \\
7 & Enrugado & Verde opaco e marrom & Forte & $12,6 \pm 1,3$ \\
8 & Enrugado & Verde opaco e marrom & Forte & $14,9 \pm 1,4$ \\
9 & Enrugado & Verde opaco e marrom & Fraco & $18,1 \pm 1,6$ \\
10 & Murcho com rugas & Verde opaco e marrom & Fraco & $20,4 \pm 1,9$ \\
11 & Murcho com rugas & Verde opaco e marrom & Fraco & $23,3 \pm 1,3$ \\
12 & Murcho sem firmeza & Verde opaco e marrom & Fraco & $26,0 \pm 2,1$ \\
13 & Murcho sem firmeza & Verde opaco e marrom & Fraco & $29,0 \pm 2,6$ \\
14 & Processo de senescência & Verde opaco e marrom & Fraco & $32,7 \pm 2,6$ \\
15 & Processo de senescência & Verde opaco e marrom & Fraco & $36,3 \pm 2,7$ \\
\hline
\end{tabular}

Figura 3. Pimentão com pele lisa, atrativa de cor verde brilhante (A), pimentão com poucas rugas de cor verde opaco (B), Pimentão enrugado de cor verde opaco e marrom (C), pimentão muito enrugado de cor verde opaco e marrom (D), Pombal, Paraíba.
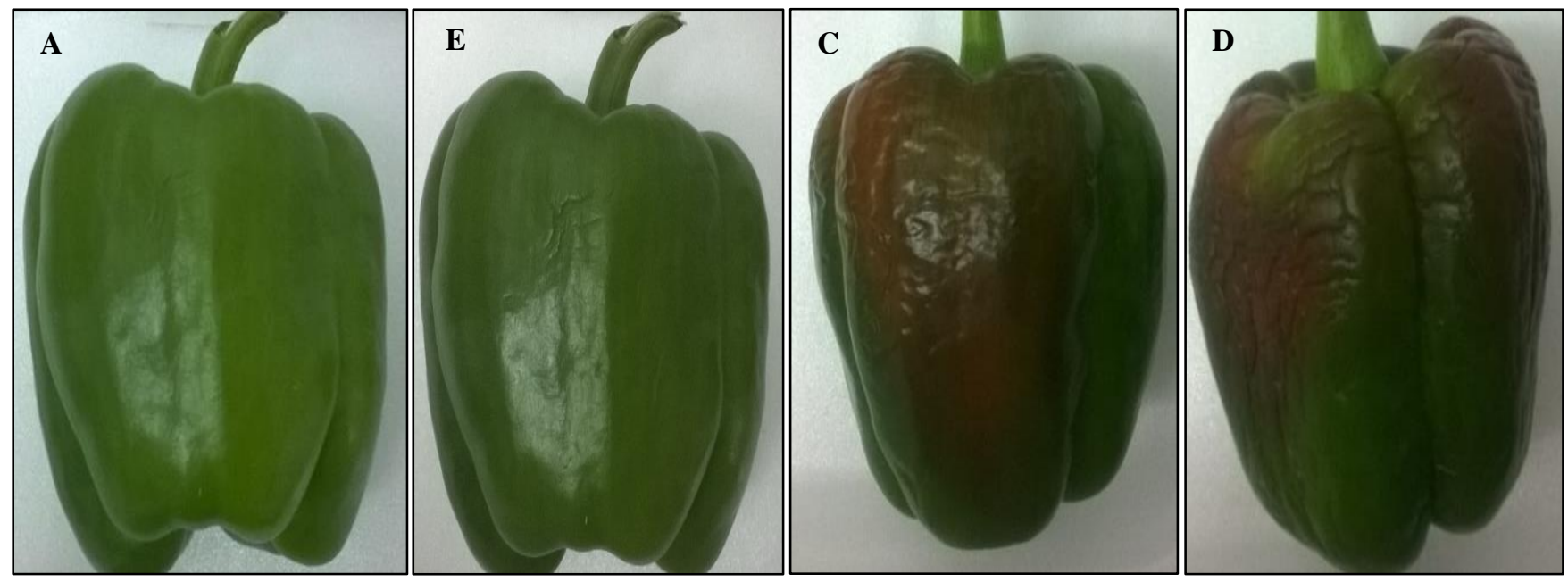

Fonte: Autor Principal (2016) 
Figura 4. Pimentão verde cortado após 15 dias de armazenamento, enrugado de cor verde opaco e marrom, Pombal, Paraíba.

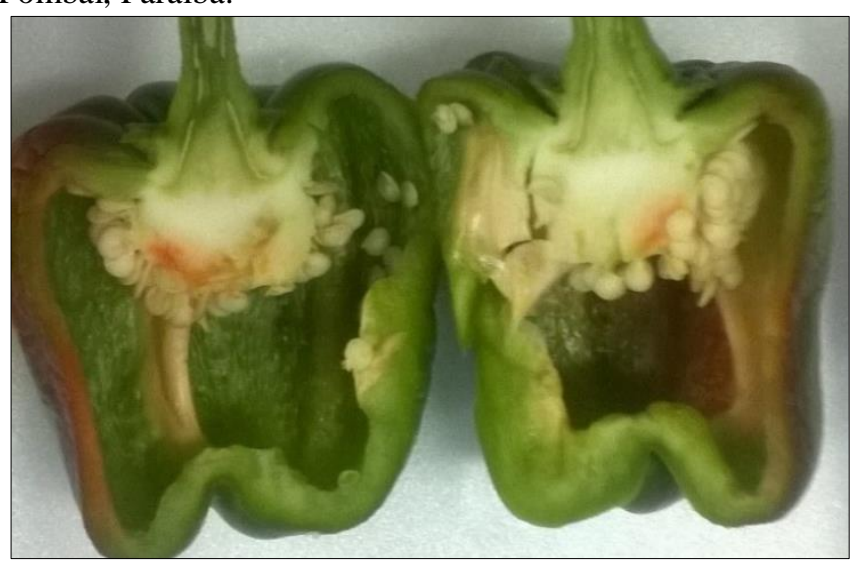

Fonte: Autor Principal (2016).

Os pimentões avaliados apresentaram cor verde brilhante até o terceiro dia de análise (Tabela 1), após esse período eles tornaram-se opacos, isso indica que os frutos sofreram perdas. De acordo com Chitarra e Chitarra (2005), a coloração é tida como o atributo de qualidade mais relevante para o consumidor. Já Ribeiro e Seravalli (2004), admite que a cor é um dos principais parâmetros que abrange a atenção do consumidor, tendo em vista que, ela é determinada pela presença de pigmentos naturais existentes nos frutos.

$\mathrm{O}$ aroma dos pimentões foram diminuindo com o passar dos dias, os frutos inicialmente possuíam um aroma forte e atrativo (Tabela 1), a partir do nono dia o aroma tornou-se mais fraco, estando os pimentões com menos atributos para um possível consumidor. De acordo com Chiappini (2008), o aroma de alguns alimentos é uma das principais características que envolve o seu consumo, sendo um atributo sensorial de grande influência durante a sua obtenção.

Houve uma perda de massa durante o armazenamento dos pimentões. Na Tabela 1 é possível identificar o comportamento crescente da perda de massa em todos os dias de armazenamento, saindo de 1,8 para 36,3\%. Lemos (2006), ao estudar a conservação pós-colheita de pimentão em condições ambientes, observou que a perda de massa em pimentões verdes num período de 20 dias foi de 32,42\%. Nota-se que o valor reportado pelo autor foi inferior ao encontrado neste trabalho, tendo em vista que, a perda de massa com 15 dias de armazenamento chegou a 36,3\%. Esse comportamento pode estar relacionado com o processo de transpiração dos pimentões.

\section{CONCLUSÕES}

Os pimentões verdes sofreram modificações visuais no decorrer do armazenamento, a estabilidade dessa hortaliça na temperatura controlada foi de aproximadamente 6 dias. A senescência do fruto foi decorrente da transpiração, o que deixou produto com características não atrativas para o consumidor.

\section{REFERÊNCIAS}

ALBIERI, S. M. J. Elaboração de material didático sobre minimamente processados: contribuição para o curso técnico em agropecuária orgânica. 2005. 142.p. Disertação (Mestrado em Educação Agrícola), Universidade Federal Rural do Rio de Janeiro, Seropédica, RJ, 2005.

ANUÁRIO BRASILEIRO DE HORTALIÇAS: Brazilian Vegetable Yearbook, Santa Cruz do Sul: Gazeta, 2012.

CANTILLANO, F. F.; GIRARD, C. L. Maçã Pós-colheita: 5 distúrbios fisiológicos. p.45-53. Disponível em: < https://www.agencia.cnptia.embrapa.br/Repositorio/5Disturbi osFisiologicosPoscolheita_000fid26cwq02 wyiv80z4s473vy2 g93v.pdf>. Acesso em 21 de agosto de 2017.

CHIAPPINI, C. C. Aromas naturais: Aromas naturais produzidos por microorganismos. Food Ingredients Brasil, n.4, p.22-24, 2008. Disponível em: <http://revistafi.com/materias/55.pdf > . Acesso em 20 de agosto de 2017.

CONAB. Companhia Nacional de Abastecimento. Boletim Hortigranjeiro, v.2, n.6, junho de 2016. Disponível em: <http://www.conab.gov.br>. Acesso em 20 de agosto de 2017.

CHITARRA, M. I. F.; CHITARRA, A. B. Pós-colheita de frutas e hortaliças: fisiologia e manuseio. Lavras: FAEPE, 2. ed. 2005,783 p.

EMBRAPA/SEBRAE. Catálogo Brasileiro de Hortaliças: saiba como plantar e aproveitar 50 das espécies mais comercializadas no País. Brasília: EMBRAPA. 2010. 59p.

FILGUEIRA, F. A. R. Solanáceas: Agrotecnologia moderna na produção de tomate, batata, pimentão, pimenta, berinjela e jiló. 1 ed. Lavras: UFLA, 2003. 333p.

LEMOS, O. L. Utilização de biofilmes comestíveis na conservação pós-colheita de pimentão. 'Magali R'. 2006. 115.p. Disertação (Mestrado em Conservação e Fitotecnia), Universidade Estadual do Sudoeste da Bahia, Bahia, 2006.

LIMA, V. L. A. G.; MÉLO, E. A.; GUERRA, N. B. Correlação entre o teor de antocianinas e caracterização cromática de polpas de diferentes genótipos de aceroleira. Brazilian Journal of Food Technology, v.10, n.1, p.51-55, 2007.

MORGADO, C. M. A.; DURIGAN, J. F.; SANCHES, J.; GALATI, V. C.; OGASSAVARA, F O. Conservação póscolheita de frutos de pimentão sob diferentes condições de armazenamento e filmes. Horticultura Brasileira, v.26, n.2, p.170-174, 2008.

MONTEIRO, M. T. M.; GOMES, V. F. F.; FILHO, P. F. M.; GUIMARÃES, F. V. A. Absorção de nutrientes por mudas de pimentão micorrizado cultivado em substrato com pó de coco. Revista Caatinga, v.22, p.95-101, 2009.

RIBEIRO, E. A.; SERAVALLI, E. A. G. Química de Alimentos. São Paulo: Edgard Blücher Ltda, 2004. 